\title{
A Small-Area Solenoid Inductor Based Digitally Controlled Oscillator
}

\author{
Hyung-Gu Park, SoYoung Kim, and Kang-Yoon Lee
}

\begin{abstract}
This paper presents a wide band, fineresolution digitally controlled oscillator (DCO) with an on-chip 3-D solenoid inductor using the $0.13 \mu \mathrm{m}$ digital CMOS process. The on-chip solenoid inductor is vertically constructed by using Metal and Via layers with a horizontal scalability. Compared to a spiral inductor, it has the advantage of occupying a small area and this is due to its 3-D structure. To control the frequency of the DCO, active capacitor and active inductor are tuned digitally. To cover the wide tuning range, a three-step coarse tuning scheme is used. In addition, the DCO gain needs to be calibrated digitally to compensate for gain variations. The DCO with solenoid inductor is fabricated in $\mathbf{0 . 1 3}$ $\mu \mathrm{m}$ process and the die area of the solenoid inductor is $0.013 \mathrm{~mm}^{2}$. The DCO tuning range is about $54 \%$ at 4.1 GHz, and the power consumption is $6.6 \mathrm{~mW}$ from a $1.2 \mathrm{~V}$ supply voltage. An effective frequency resolution is $0.14 \mathrm{kHz}$. The measured phase noise of the DCO output at $5.195 \mathrm{GHz}$ is $-110.61 \mathrm{dBc} / \mathrm{Hz}$ at 1 MHz offset.
\end{abstract}

Index Terms-Solenoid inductor, active capacitor, wide tuning range, automatic three-step coarse tuning, fine-resolution, DCO

Manuscript received Sep. 19, 2012; accepted Dec. 22, 2012.

College of Information and Communication Engineering, Sungkyunkwan University, Suwon, Korea

E-mail : klee@skku.edu

\section{INTRODUCTION}

The DCO (digitally controlled oscillator) is one of the most critical blocks in the digital PLL. Spiral inductors based on the RF process have been used in the DCOs. They occupy a large area and thereby, increasing the process cost. A solenoid inductor implementation has been studied in [1] and it mainly uses MEMS technology. By virtue of good conductivity of copper, the copper electroplated inductor has a high quality factor and it has an inductance of a tenth of $\mathrm{nH}$ [2]. However, post processing of the MEMS method requires an additional process cost. As the CMOS technology has started to adopt $\mathrm{Cu}$ instead of $\mathrm{Al}$ for its metal interconnection below the $0.13 \mu \mathrm{m}$ process, it opens the possibility of implementing a low series resistance inductor in an LC DCO. However, planar spiral inductors are still being used for LC DCOs in the digital CMOS process. In this paper, a solenoid inductor using Metal stack is proposed to replace the spiral inductors which require RF CMOS process.

To overcome the limitation of the varactor capacitance, a sigma-delta modulator is used to implement the fine capacitance [3]. In this case, the dithering bits of the sigma-delta modulator need to be increased in order to implement a finer resolution. Therefore, the area and power consumption are also increased. In this paper, an active capacitor and inductor are used for the frequency control, overcoming the minimum frequency resolution problem.

Several techniques are used to cover the wide frequency range with a single LC-oscillator. The automatic coarse tuning scheme is typically used to widen the tuning range. The variation of the DCO gain (KDCO) is large in wide range applications. The automatic calibration of the DCO 
gain is necessary to guarantee the same loop bandwidth for the phase noise and lock-time performances regardless of the channel frequency.

This paper presents a wide band, fine-resolution DCO with an on-chip 3-D solenoid inductor using an automatic three-step coarse tuning loop and gain tuning loop. Electrical characteristics of solenoid inductor are evaluated in terms of inductance and its quality factor by using 3DEM simulation in order to reduce the LC DCO area in the digital CMOS process.

Section II shows the structure of the on-chip solenoid inductor and Section III explains wide range DCO with on-chip solenoid inductor. Section IV shows the measurement results of the proposed DCO. Section V concludes this paper.

\section{ON-CHIP SOLENOID INDUCTOR}

Generally, a spiral structure is fabricated by using the planar CMOS process. On the other hand, the solenoid inductor shown in Fig. 1(a) is built by using Metal and Via interconnection. Moreover, the axis of the inductor is parallel to the substrate. This makes it less susceptible to substrate losses. In the structure shown in Fig. 1(b), the bottom plates are represented by M1 and the top plates by M6. Posts are connected between the top plates and the bottom plates by Vias (V1-V5). The solenoid inductance is determined by the number of turns $(\mathrm{N})$, the cross-sectional area (A) that the magnetic flux is crossing, and the pitch between the turns $(\mathrm{P})$. The cross sectional area $(\mathrm{A})$ is given as $\mathrm{W} \times \mathrm{H}$ and the height is fixed by the process. Also, inductance is proportional to the width (W) and the number of turns $(\mathrm{N})$.

To estimate the exact performance, when the solenoid is built into an LC DCO, a 3-D finite element electromagnetic (EM) simulator has been used to perform a precise analysis of the proposed solenoid inductor.

Fig. 2 shows the simulated inductance of the solenoid inductor with respect to the number of turns $(\mathrm{N})$. The inductance values are proportional to the number of turns.

Fig. 3 shows the simulated inductance of the solenoid inductor with respect to the space between turns $(\mathrm{N})$. The inductance values are inverse proportional to the space between turns.

Fig. 4 shows the simulated inductance of the solenoid inductor with respect to the metal width (W). The

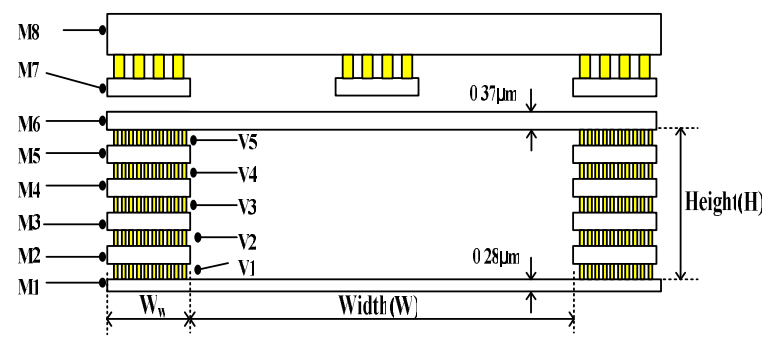

(a)

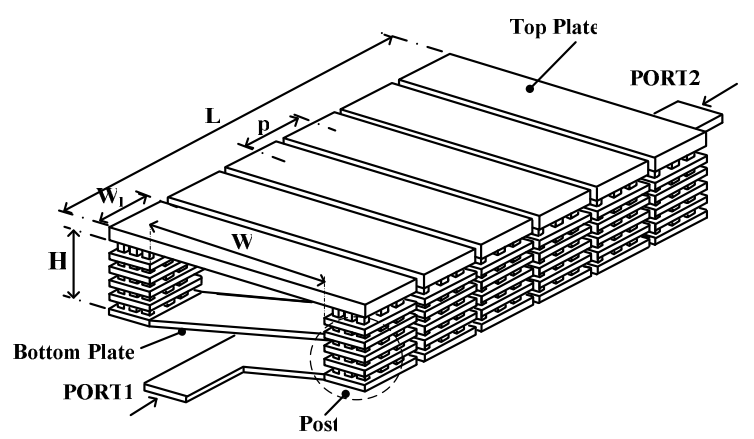

(b)

Fig. 1. (a) Cross sectional view, (b) top view of solenoid inductor.

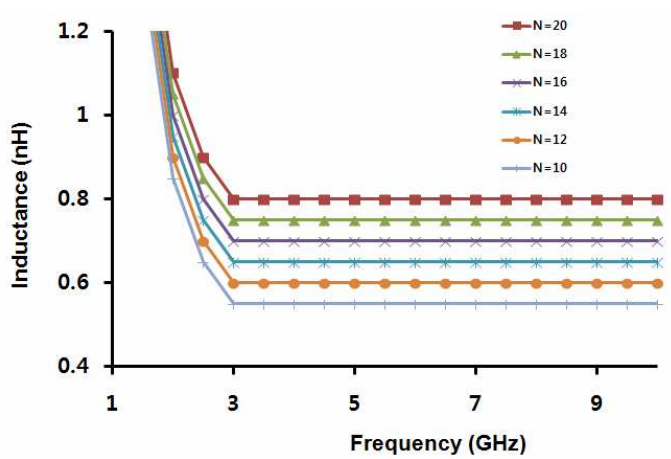

Fig. 2. Simulated inductance of the solenoid inductor with respect to the number of turns $(\mathrm{N})$.

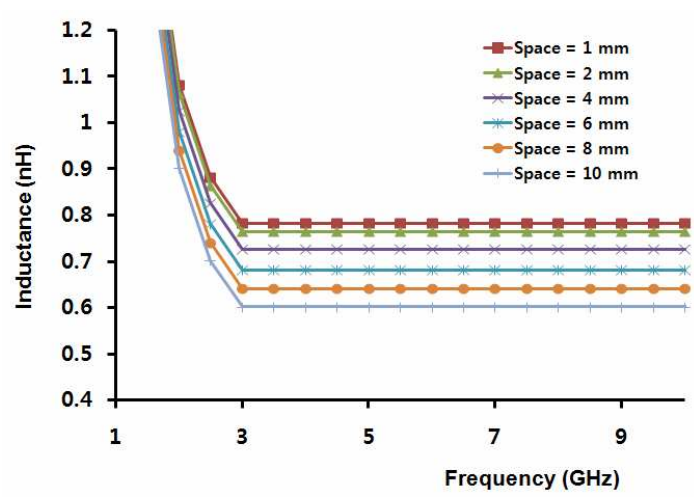

Fig. 3. Simulated inductance of the solenoid inductor with respect to the space between turns. 


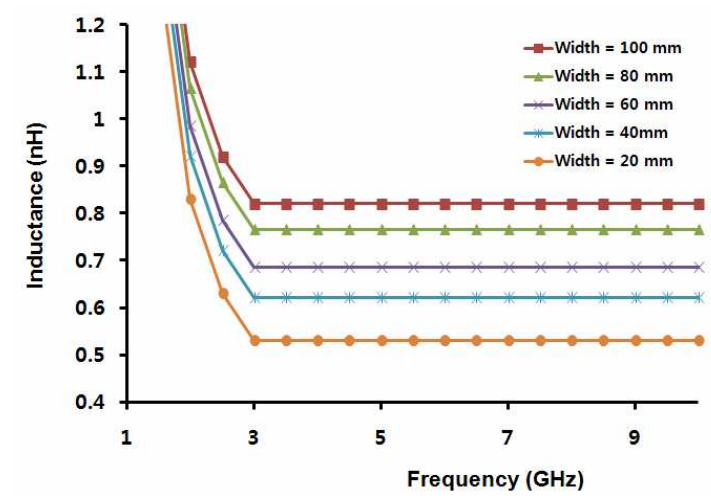

Fig. 4. Simulated inductance of the solenoid inductor with respect to the metal width (W).

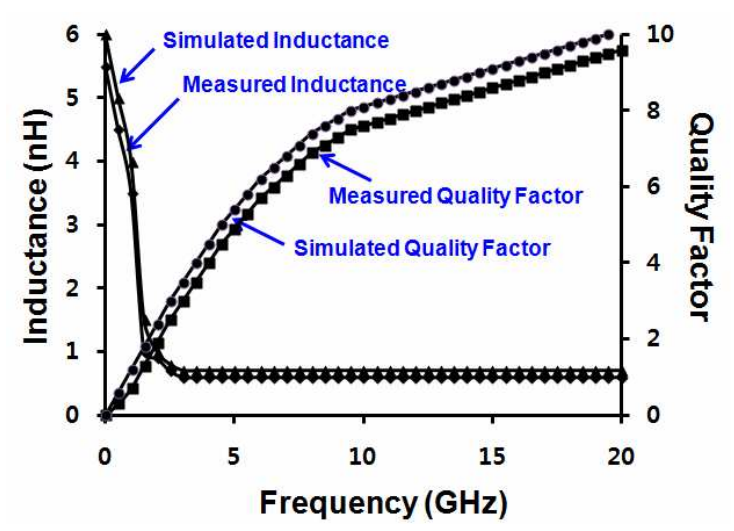

Fig. 5. Inductance and quality factor of the solenoid inductor.

inductance values are proportional to the metal width.

The measured inductance and the quality factor from de-embedding are shown along with the simulation results in Fig. 5. Both the simulated and the measured inductances are almost the same from 0.6 to $0.9 \mathrm{nH}$ and it is as shown in Fig. 5. The measured quality factor is lower than that of the simulated result as shown in Fig. 5. However, the two results are almost the same over the range of interest around $5 \mathrm{GHz}$. Measurement results still show that the self-resonance frequency (SRF) is over $20 \mathrm{GHz}$.

Fig. 6 shows the quality factors of the solenoid inductor and the spiral inductor when the inductance values are both $0.6 \mathrm{nH}$. The highest thick metal is not used to form the solenoid inductor, while it is used in the spiral inductor. The quality factors of the solenoid inductor and the spiral inductor are around 6 and 12 at the frequency of $5 \mathrm{GHz}$, respectively.

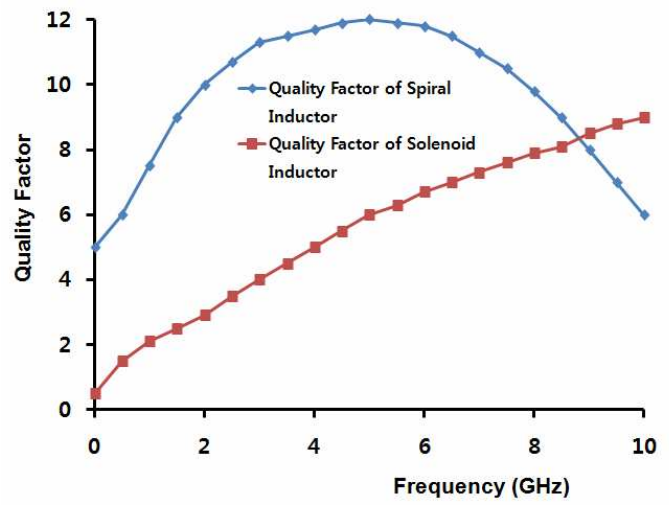

Fig. 6. Quality factors of the solenoid inductor and spiral inductor.

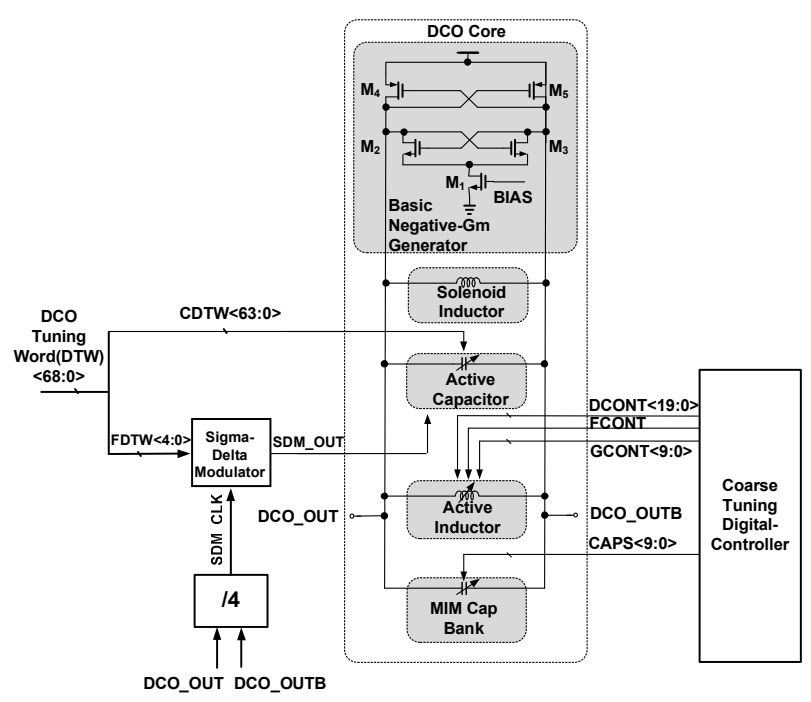

Fig. 7. The block diagram of the proposed DCO with a solenoid inductor.

\section{WIDE RANGE DCO WITH ON-ChIP SOLENOID INDUCTOR}

Fig. 7 shows the block diagram of the proposed DCO with an on-chip solenoid inductor. It is composed of the DCO core, a sigma-delta modulator, and a coarse tuning digital controller. A $3^{\text {rd }}$ order MASH type sigma-delta modulator is used with five dithering bits (FDTW $<4: 0>$ ).

The DCO Core consists of a solenoid inductor, an active inductor, and an active capacitor, a MIM cap bank, and a negative-Gm generator. The on-chip solenoid inductor shown in Fig. 1 is used to provide the fixed inductance value and meet the phase noise performance requirements. The active inductor is used for the wide-frequency range tuning and narrow-frequency range tuning control. The 
cap bank, which is composed of switches and MIM capacitors, provides the mid-frequency tuning range of the DCO. The quality factor can be increased resulting in the improvement of the phase noise performance by using the solenoid inductor in parallel with the active inductor.

Fig. 8 shows the active capacitor circuit and $\mathrm{C}-\mathrm{V}$ characteristic graph. Active capacitor is also used for the fine frequency tuning. Fine capacitance can be effectively implemented by using the capacitance difference in accumulation mode and inversion mode of active capacitor [4].

When the oscillation amplitude is $\mathrm{A}_{0}$, the total capacitance $(\Delta \mathrm{C})$ is given by Eq. (1)

$$
\Delta \mathrm{C}=\left(\mathrm{C}_{\mathrm{A}}+\mathrm{C}_{\mathrm{B}}\right)-\left(\mathrm{C}_{\mathrm{C}}+\mathrm{C}_{\mathrm{D}}\right)
$$

where, $C_{A}$ and $C_{D}$ are capacitance in inversion mode, $C_{B}$ and $\mathrm{C}_{\mathrm{C}}$ are capacitance in accumulation mode.

Input tuning code word, $\mathrm{CDTW}<63: 0>$, finely controls the frequency of the DCO by adjusting the value of active capacitance. Also, the dithering output (SDM_O(2:0)) of second-order sigma-delta modulator (SDM) is applied to the Active Cap in order to increase the resolution of the DCO.

Coarse tuning controller in Fig. 7 selects the optimum capacitance values, CAPS $<9: 0>$, to cover the target frequency closely against the PVT variations of the inductor or capacitances. In addition, active inductor control bits, DCONT $<19: 0>$, adjust the frequency of the DCO by the middle frequency amount between the wide

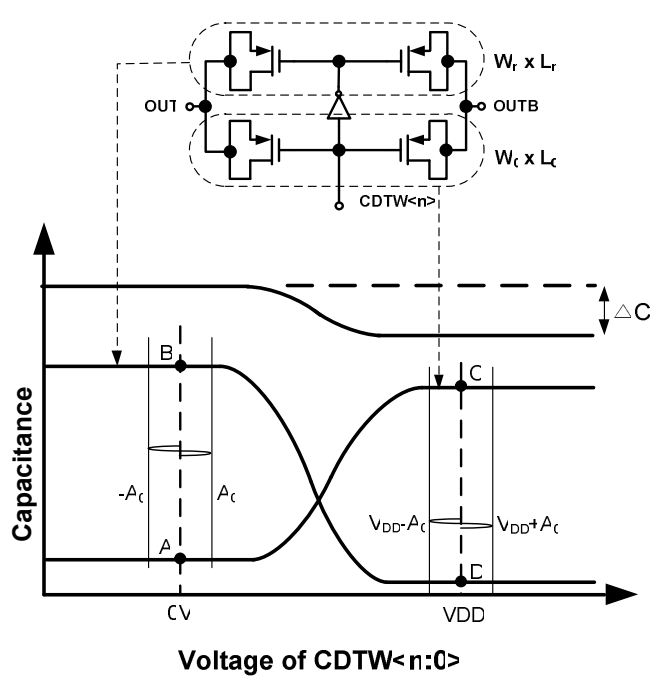

Fig. 8. The schematic of the proposed the active capacitor. range coarse tuning and fine tuning.

Fig. 9 shows the schematic of the active inductor. It is a differentially configured gyrator-C active inductor [5]. The inductance $\left(L_{e q}\right)$ of the active inductor can be tuned by varying $G_{d s}, G_{d s}, \mathrm{G}_{d s} 9, G_{d s 11}$, and $G_{d s G C B}$, where $G_{d s 5}, G_{d s} 7$, $G_{d s}$ and $G_{d s 11}$ are the drain conductances of $\mathrm{M}_{5}, \mathrm{M}_{7}, \mathrm{M}_{9}$ and $\mathrm{M}_{11}$ in Fig. 9, respectively. $G_{d s G C B}$ is the $\operatorname{PMOS}\left(\mathrm{M}_{1}\right)$ drain conductance of the DCO gain control cell in the DCO gain control bank in Fig. 10 and $G_{d s}$ is controlled by $I_{D 7}$. As mentioned above, $G_{d s} 9, G_{d s 11}$, and $G_{d s G C B}$ are also controlled by $I_{D 9}, I_{D 11}$, and $I_{D G C B}$, respectively. $I_{D}$ is dependent on the gate voltage and the size of the MOS (W/L). Among transistors in Active Ind Control Block of Fig. 9, $\mathrm{M}_{9}$ has the largest size for the wide tuning range, $\mathrm{M}_{11}$ has the medium size for the narrow tuning range, and $\mathrm{M}_{7}$ has the smallest size for the fine tuning step.

Thus, the method for the inductance tuning is to control the drain conductance $G_{d s}, G_{d s}, G_{d s l l}$, and $G_{d s G C B}$ except $G_{d s 5}$ by the gate voltage and the size of the MOS. $I_{D 7}, I_{D 9}$, and $I_{D 11}$ are controlled by the FCDTW $<64: 0>$, FCONT and DCONT $<19: 0>$ in the active inductor control bank, respectively. $I_{D G C B}$ is controlled by the DCONT $<19: 0>$ in the DCO gain control bank.

Fig. 10 shows the complete schematic of the gain control bank of the proposed DCO. It is composed of the ten DCO gain control cell arrays which are controlled by the signals from the GCONT $<9: 0>$. When the GCONT $<\mathrm{N}>$ is HIGH, the gate of the PMOS $\left(\mathrm{M}_{1}, \mathrm{M}_{2}\right)$ is connected to the FCDTW $<64: 0>$. However, if the GCONT $<\mathrm{N}>$ is LOW, the gate of PMOS $\left(\mathrm{M}_{1}, \mathrm{M}_{2}\right)$ is connected to the VDD to disable the corresponding DCO gain control cell. The switches in the gain control cell are connected to the gate of the PMOS in order to reduce the degradation of the parasitic capacitance of the switches.

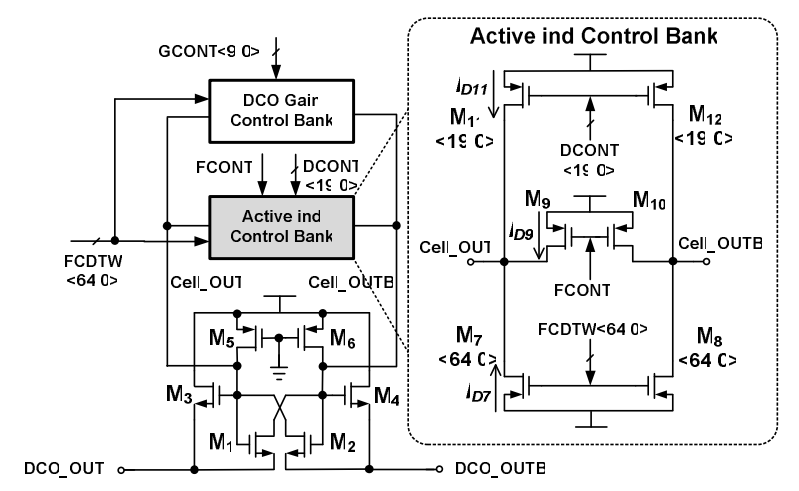

Fig. 9. The schematic of the proposed the active inductor. 


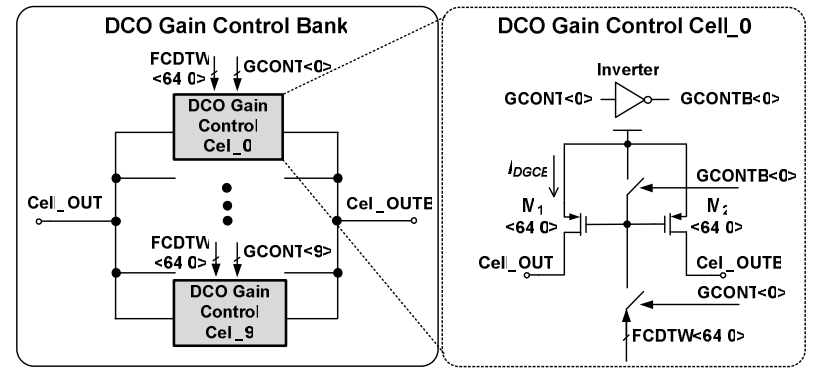

Fig. 10. The schematic of the DCO gain control bank.

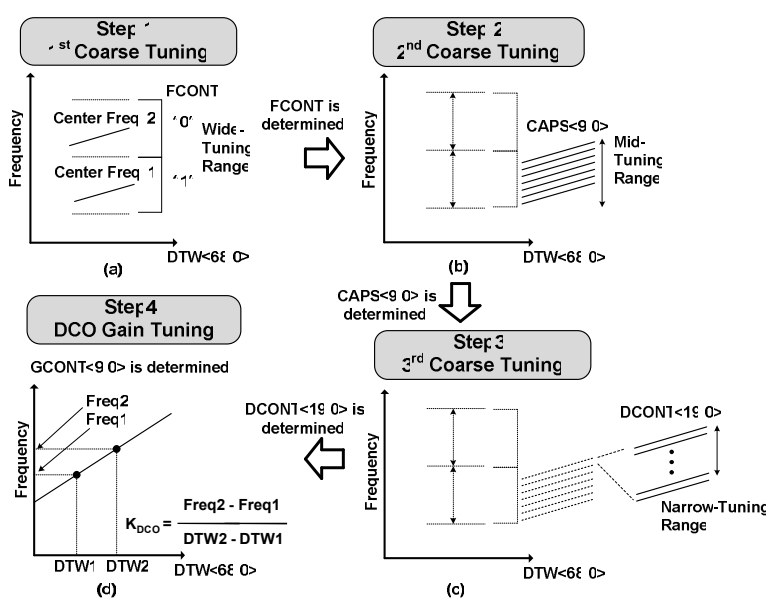

Fig. 11. The concept of proposed three-step coarse and gain tuning.

The automatic three-step coarse and gain tuning procedure are as follows. Its principle is shown in Figs. 11(a)-(d).

Step 1: The $1^{\text {st }}$ coarse tuning is the wide-frequency range tuning. The DCO frequency is measured through the coarse tuning digital-controller, as seen in Fig. 11. The optimum center frequency is selected by the FCONT adjusting the large value inductance of the active inductor as shown in Fig. 11(a).

Step 2: The $2^{\text {nd }}$ coarse tuning is the mid-frequency range tuning. The optimum MIM capacitances are selected through the $2^{\text {nd }}$ coarse tuning process after the $1^{\text {st }}$ coarse tuning is completed as shown in Fig. 11(b).

Step 3: The 3rd coarse tuning is the narrow-frequency range tuning. Because the difference between the frequency curves at the $2^{\text {nd }}$ coarse tuning is several $\mathrm{MHz}$, additional tuning is required. The tuning procedure is processed by adjusting the inductance of the active inductor as shown in Fig. 11(c).

Step 4: The DCO gain tuning begins when the threestep coarse tuning is completed. When the frequency of the $\mathrm{DCO}$ is high in the tuning range, the $\mathrm{DCO}$ gain $\left(\mathrm{K}_{\mathrm{DCO}}\right)$ is higher than that of the center frequency. Thus, the DCO gain should be decreased by increasing the number of active gain control block in Fig. 10. On the other hand, when the frequency of the DCO is low in the tuning range, the DCO gain should be increased by decreasing the number of active gain control block in Fig. 10.

The $\mathrm{K}_{\mathrm{DCO}}$, which is defined as the frequency deviation of the DCO with respect to a 1-LSB change, is equal to the frequency resolution. Thus, the $\mathrm{K}_{\mathrm{DCO}}$ is controlled by the value of the signal, GCONT $<9: 0>$, which adjust the frequency resolution.

As shown in Fig. 11(d), the $\mathrm{K}_{\mathrm{DCO}}$ can be estimated by dividing the difference of Freq 2 and Freq1 by the difference of DTW2 and DTW1. It is measured and calculated though the coarse tuning digital-controller. And then GCONT $<9: 0>$ are adjusted so the $\mathrm{K}_{\mathrm{DCO}}$ can reach its reference boundary.

Fig. 12 shows the automatic three-step coarse and gain tuning loop. When frequency tuning range of the DCO is wide, the variation of the $\mathrm{DCO}$ gain $\left(\mathrm{K}_{\mathrm{DCO}}\right)$ is very large depending on the frequency. Thus, the DCO gain control bank in Fig. 9 is required for the automatic DCO gain tuning loop. The $\mathrm{K}_{\mathrm{DCO}}$ can be calculated with 15-bit counter and digital blocks that can be shared with the three-step coarse tuning block. The frequency of DCO is detected through the 15-bit counter and is compared with the reference value. In the each step coarse and gain tuning control signals (FCONT, CAPS $<9: 0>$, DCONT $<19: 0>$,

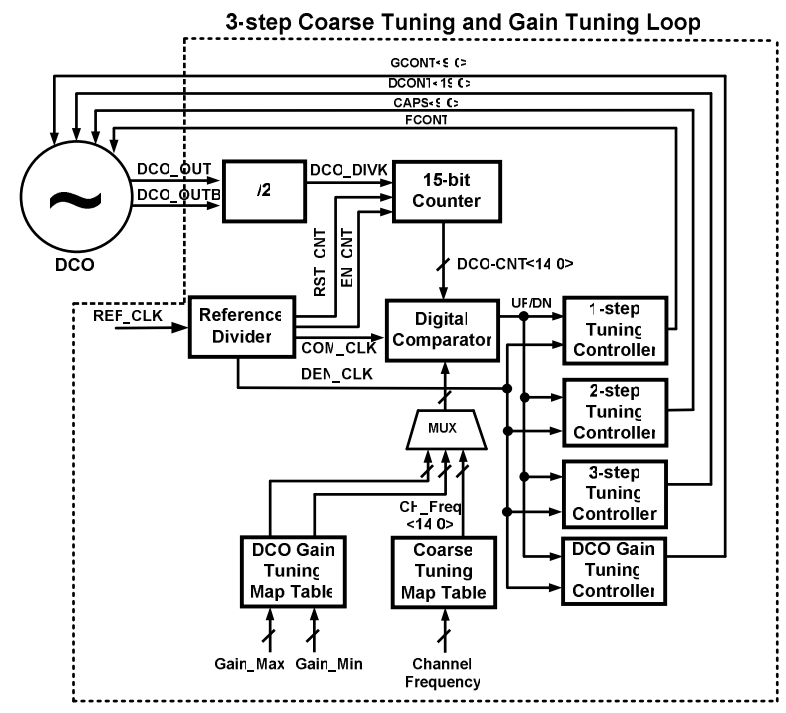

Fig. 12. The automatic three-step coarse and gain tuning loop of the proposed DCO. 
and GCONT $<9: 0>$ ) are determined digitally based on the comparison result. The phase difference between the external reference clock and DCO is detected at the phase frequency detector in the all-digital PLL (ADPLL). Then, it is converted into the digital tuning word, DTW $<68: 0>$, through the digital filter. The DTW $<68: 0>$ is adjusted to finely tune the phase and frequency of the DCO at the fine tuning stage after the three-step coarse and gain tuning.

\section{EXPERIMENTAL RESULTS}

This chip is fabricated using the digital CMOS process with $0.13 \mu \mathrm{m}$ technology, a single poly layer, six layers of metal, the option of metal-insulator-metal (MIM) capacitors, and high sheet resistance poly resistors. Die area of the solenoid inductor and DCO are $0.013 \mathrm{~mm}^{2}$ and $0.034 \mathrm{~mm}^{2}$, respectively. Fig. 13 shows the chip microphotograph of the DCO.

The LC DCO with the on-chip solenoid inductor ( $\mathrm{W}=60$ $\mu \mathrm{m}, \mathrm{N}=18$ ) is measured using a spectrum analyzer (HP E4440A).

Table 1 shows the measured tuning range and step of the DCO. The frequency tuning range that can be achieved with only capacitance tuning is $1.2 \mathrm{GHz}$. As a three-step

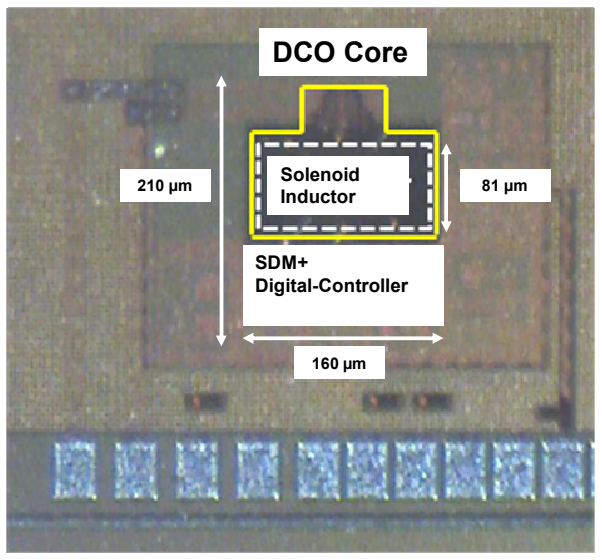

Fig. 13. Chip microphotograph.

Table 1. Measured Tuning Range and Step of the DCO.

\begin{tabular}{|c|c|c|c|c|}
\hline & $\begin{array}{c}1 \text { st Coarse } \\
\text { Tuning }\end{array}$ & $\begin{array}{c}\text { 2nd Coarse } \\
\text { Tuning }\end{array}$ & $\begin{array}{c}\text { 3rd Coarse } \\
\text { Tuning }\end{array}$ & $\begin{array}{c}\text { Fine Tuning } \\
\text { (nteger/Fractional) }\end{array}$ \\
\hline $\begin{array}{c}\text { Control } \\
\text { Signal }\end{array}$ & FCONT & CAPS $<9: 0>$ & $\begin{array}{c}\text { DCONT } \\
<9: 0>\end{array}$ & $\begin{array}{c}\text { CDTW }<63: 0>/ \\
\text { FDTW }<4: 0>\end{array}$ \\
\hline $\begin{array}{c}\text { Tuning } \\
\text { Range }\end{array}$ & $2.2 \mathrm{GHz}$ & $1.2 \mathrm{GHz}$ & $2.7 \mathrm{MHz}$ & $\begin{array}{c}294 \mathrm{kHz} / \\
4.6 \mathrm{kHz}\end{array}$ \\
\hline $\begin{array}{c}\text { Tuning } \\
\text { Step }\end{array}$ & $1.1 \mathrm{GHz}$ & $1.2 \mathrm{MHz}$ & $2.6 \mathrm{kHz}$ & $\begin{array}{c}4.6 \mathrm{kHz} / \\
0.14 \mathrm{kHz}\end{array}$ \\
\hline
\end{tabular}

coarse tuning scheme is used, the tuning range can be widened by $1 \mathrm{GHz}$ under the same capacitance value. This has the effect of widening the tuning range without using extra capacitance. As a result, measured total tuning range is about $54 \%$ by coarse and fine tuning from $3 \mathrm{GHz}$ to 5.2 GHz.

Figs. 14(a) and (b) show the transient simulation results of the DCO when the output frequency is $3.0 \mathrm{GHz}$ and 5.2 $\mathrm{GHz}$, respectively. The voltage swing is reduced as the output frequency is lower because the effective capacitance at the output of the DCO is increased. Thus, DCO current is controlled by the BIAS voltage in the DCO core in order to guarantee the oscillation in the wide range.

Fig. 15 shows the measured output spectrum of the DCO when the output frequency is $5.15 \mathrm{GHz}$. Its power level is around $-17 \mathrm{dBm}$ when measured through the test buffer inside the chip.

Fig. 16 shows the PVT corner simulation results of the DCO. The typical condition for the DCO is that Process, Voltage, and Temperature are TT, $1.2 \mathrm{~V}$, and $27^{\circ} \mathrm{C}$, respectively. The voltage swing of the DCO at the slow corner condition (SS, $1.08 \mathrm{~V}, 27^{\circ} \mathrm{C}$ ) is reduced compared with that at the typical condition. On the other hand, the voltage swing at the fast corner condition (FF, $1.32 \mathrm{~V}$, -

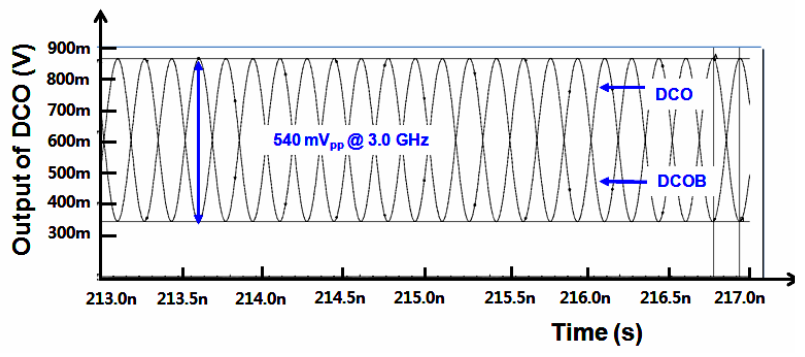

(a)

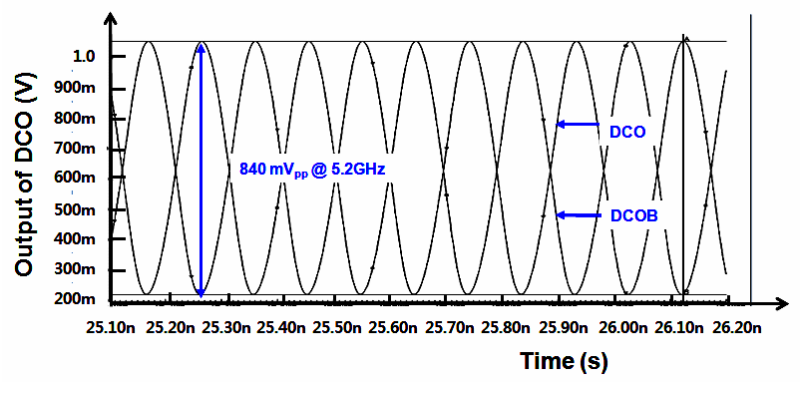

(b)

Fig. 14. Transient simulation results of the DCO when the output frequency is (a) $3.0 \mathrm{GHz}$, (b) $5.2 \mathrm{GHz}$. 


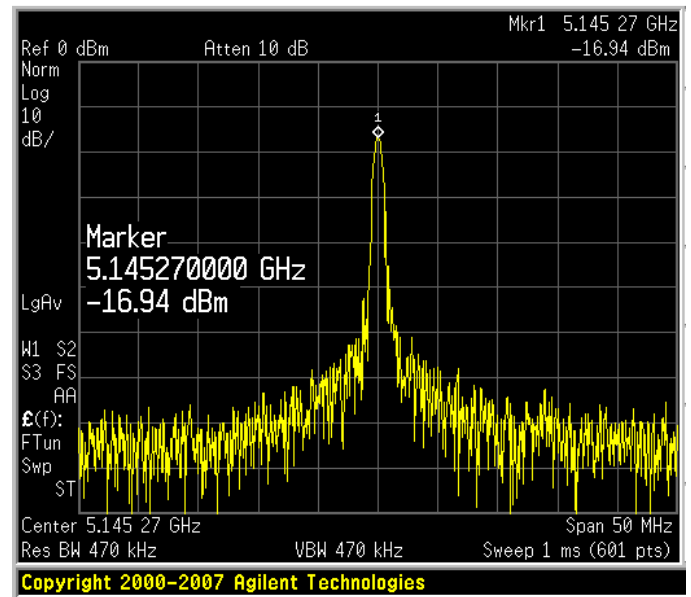

Fig. 15. Measured spectrum of the DCO.

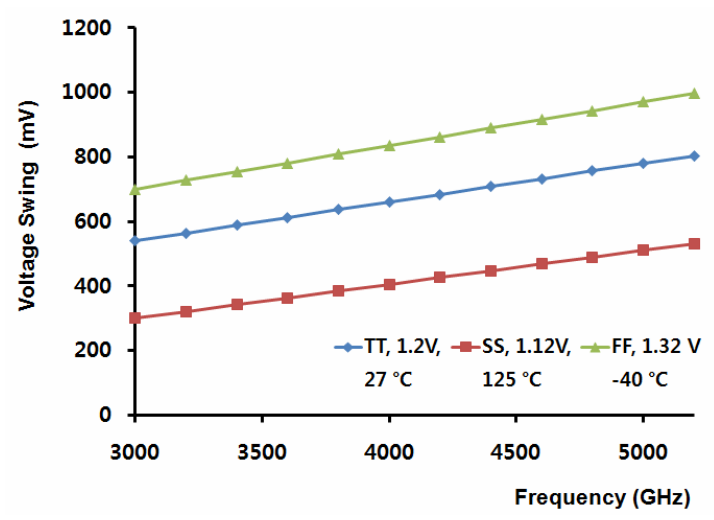

Fig. 16. PVT corner simulation results of the DCO.

$40{ }^{\circ} \mathrm{C}$ ) is increased compared with that at the typical condition. The BIAS level in the DCO Core can be controlled in order to adjust the voltage swing at corner cases.

The frequency resolution for the 1-LSB of the CDTW $<63: 0>$ is $4.6 \mathrm{kHz}$. Thus, the effective timeaveraged frequency resolution done by the 5-bit SDM can be calculated as Eq. (2)

$$
\Delta f^{\Delta \Sigma}=4.6 \mathrm{kHz} / 2^{5}=0.14 \mathrm{kHz}
$$

From (2), the $\mathrm{K}_{\mathrm{DCO}}$ is about $0.14 \mathrm{kHz} / \mathrm{LSB}$ because the frequency resolution for the 1 -LSB of the DTW $<68: 0>$ is $0.14 \mathrm{kHz}$.

Fig. 17 shows that the measured phase noise is -110.61 $\mathrm{dBc} / \mathrm{Hz}$ at $1 \mathrm{MHz}$ offset from the carrier.

The $\mathrm{FoM}_{T}$ (figure of merit with the frequency tuning range) for the DCO can be calculated using Eq. (3).

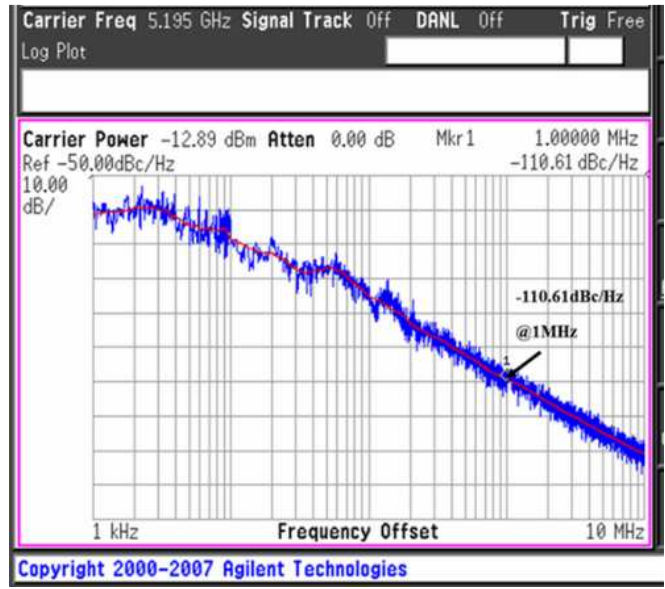

Fig. 17. The measured DCO phase noise.

Table 2. Performance Summary of the DCO.

\begin{tabular}{|c|c|c|c|c|}
\hline Parameters & {$[3]$} & {$[6]$} & {$[7]$} & This work \\
\hline Process & $\begin{array}{c}0.13 \mu \mathrm{m} \\
\text { CMOS }\end{array}$ & $\begin{array}{c}0.18 \mu \mathrm{m} \\
\text { CMOS }\end{array}$ & $\begin{array}{c}65 \mathrm{~nm} \\
\text { CMOS }\end{array}$ & $\begin{array}{c}0.13 \mu \mathrm{m} \\
\text { CMOS }\end{array}$ \\
\hline Inductor Type & Sprial & Sprial & Sprial & Solenoid \\
\hline DCO Area (mm $\left.{ }^{2}\right)$ & 0.073 & N/A & 0.32 & 0.034 \\
\hline Supply Voltage (V) & 1.5 & 1.8 & 1.8 & 1.2 \\
\hline $\begin{array}{c}\text { Power Consumption } \\
(\mathrm{mW})\end{array}$ & 3.45 & 5 & 28.8 & 6.6 \\
\hline $\begin{array}{c}\text { Center Frequency } \\
(\mathrm{GHz})\end{array}$ & 2.4 & 3.8 & 3 & 4.1 \\
\hline Tuning Range (\%) & 20.8 & 26.3 & 26 & 54 \\
\hline $\begin{array}{c}\text { Frequency } \\
\text { Resolution (kHz) }\end{array}$ & 0.718 & 20 & 0.15 & 0.14 \\
\hline $\begin{array}{c}\text { Phase Noise } \\
\text { @ 1 MHz ( dBc/Hz) }\end{array}$ & -117.0 & -123 & -127.5 & -110.61 \\
\hline FoM $\mathrm{T}(\mathrm{dBc} / \mathrm{Hz})$ & -185.6 & -194.4 & -190.2 & -193.1 \\
\hline
\end{tabular}

$F O M_{T}=P N\left(f_{\text {offset }}\right)-20 \log \left(\frac{f_{o}}{f_{\text {offset }}}\right)+10 \log \left(\frac{P_{D C}}{1 m W}\right)-20 \log \left(\frac{F D R}{10}\right)$

where $f_{\text {offse }}$ is the offset frequency, $f_{o}$ is the oscillation frequency, $P N\left(f_{\text {offset }}\right)$ is the phase noise at the $f_{\text {offset }}, P_{D C}$ is the DC power consumption and the FDR is the frequency tuning range in a percentage.

The performance of the proposed DCO is summarized in Table 2.

The $\mathrm{FoM}_{T}$ of this work is smallest except [6], which adopted the spiral inductor for the LC DCO core, respectively. As can be seen in Fig. 6, the quality factor of the solenoid inductor is smaller than that of the spiral inductor. Also, it is smaller than that of the active inductor in [5]. Thus, the phase noise performance of this work is inferior to those of [5] and [6]. According to our 
simulation, the phase noise of the DCO with the proposed solenoid inductor at the output frequency of $5 \mathrm{GHz}$ is degraded by about $4 \mathrm{~dB}$ and $2 \mathrm{~dB}$ compared with those using the spiral inductor and active inductor, respectively. However, the main target of this work is to reduce the die size with small area solenoid inductor. The phase noise performance can be further improved with more die area. The proposed DCO has the smallest size thanks to the proposed solenoid inductor with the small area.

\section{ConClusions}

A wide band, small-area DCO with an solenoid inductor using an automatic three-step coarse and gain tuning loop is fabricated using the $0.13 \mu \mathrm{m}$ digital CMOS process without any additional RF options. Due to the Metal stacked solenoid inductor, the chip area is remarkably reduced compared to those using a conventional spiral inductor. The tuning range of the DCO is $3 \mathrm{GHz}-5.2 \mathrm{GHz}$ with the effective frequency resolution of $0.14 \mathrm{kHz}$. The power consumption is $6.6 \mathrm{~mW}$ from a $1.2 \mathrm{~V}$ supply. The phase noise of the DCO output at $5.195 \mathrm{GHz}$ is -110.61 $\mathrm{dBc} / \mathrm{Hz}$ at $1 \mathrm{MHz}$ offset. Moreover, this small area LC DCO is suitable for low cost wireless PLLs.

\section{ACKNOWLEDGMENTS}

This work was supported by the National Research Foundation of Korea (NRF) grant funded by the Korea government (MEST) (No. 2011-0027658).

\section{REFERENCES}

[1] R. B. Staszewski, P. T. Balsara, "Phase-domain alldigital phase-locked loop," IEEE Trans. Circuits Syst. II, Expr. Briefs, vol. 52, no. 3, pp. 159-163, Mar. 2005.

[2] P.-L. Chen, C.-C. Chung and C.-Y. Lee, "A portable Digitally Controlled Oscillator Using Novel Varactors," IEEE Trans. on Circuits and Systems, Vol. 52, No. 5, May 2005.

[3] R. B. Staszewski, D. Leipold, K. Muhammad, and P. T. Balsara, "Digitally controlled oscillator (DCO)-based architecture for RF frequency synthesis in a deep-submicrometer CMOS process," IEEE Trans. Circuits Syst. II, vol. 50, no.
11, pp. 815-828, Nov. 2003.

[4] S.-S. Yoo, Y.-C. Choi, H.-J. Song, S.-C. Park, J.-H. Park, and H.-J. Yoo, "A 5.8-GHz High-Frequency Resolution Digitally Controlled Oscillator Using the Difference Between Inversion and Accumulation Mode Capacitance of pMOS Varactors," IEEE Transactions on Microwave Theory and Techniques, vol. 59, no. 2, pp. 375-382, Feb. 2011.

[5] L. Lu, H. Hsieh, and Y. Liao, "A Wide TuningRange CMOS VCO With a Differential Tunable Active Inductor," IEEE Trans. Microwave Theory Tech., vol. 3, pp. 3462-3468, Sept. 2006.

[6] S. Wang, J. Quan, R. Luo, and H. Cheng, "A noise reduced digitally controlled oscillator using complementary varactor pair," Proceedings of IEEE International Symposium on Circuits and Systems, pp. 937-940, May 2007.

[7] L. Fanori, A. Liscidini, and R. Castello, "Capacitive Degeneration in LC-Tank Oscillator for DCO Fine-Frequency Tuning," IEEE Journal of Solid-State Circuits, vol. 45, no. 12, pp. 2737-2745, December 2010.

[8] J. B. Yoon, B. K. Kim, C. H. Han, E. Yoon, and C. K. Kim, "Surface micromachined solenoid on-Si and on-galss inductor for RF applications," IEEE Electron Device Lett., vol. 20, no. 9, pp. 487-489, Sep 1999.

[9] S.-H. Seok, C. Nam, W.-S. Choi, K.-J. Chun, “A High Performance Solenoid-type MEMS Inductor," Journal of semiconductor technology and science, vol. 1, No. 3, June, 2001.

[10] T. H. Lee, "The Design of CMOS Radio-frequency integrated Circuits," pp.50, Cambridge, United Kingdom, 1998.

[11] C. Nam, J.-S. park, Y.-G. Pu, K.-Y. Lee, “A 1-GHz Tuning range DCO with a $3.9 \mathrm{KHz}$ discrete tuning step for UWB frequency Synthesizer," IEICE Trans. on Electronics, E93-C, No. 6, pp. 770-776, Jun. 2010.

[12] B. Soltanian, H. Ainspan, W. Rhee, D. Friedman, P.Kinget, "An Ultra Compact Differentially Tuned $6 \mathrm{GHz}$ CMOS LC VCO with Dynamic CommonMode Feedback," Proceedings of Custom Integrated Circuits Conference, pp 671-674,2006.

[13] R. Tao and M. Berroth, "The design of $5 \mathrm{GHz}$ voltage controlled ring oscillator using source 
capacitively coupled current amplifier," IEEE Radio Frequency Integrated Circuits Symposium, pp. 623-626, 2003.

[14] H. R. Rategh,, H. Samavati, and T. H. Lee, "A $5 \mathrm{GHz} 32 \mathrm{~mW}$ CMOS Frequency Synthesizer with an Injection Locked Frequency Divider," Proceedings of Symposium on VLSI Circuits, pp. 671-674, 1999.

[15] N. H. W. Fong, J.-O. Plouchart, N. Zamdmer, D. Liu, L.F. Wagner, C. Plett, and N. G. Tarr, "A 1-V 3.8-5.7 GHz wideband VCO with differentially tuned accumulation MOS varactor for commonmode noise rejection in CMOS SOI technology," IEEE Transactions on Microwave Theory and Techniques, vol. 51, no. 8, pp. 1952-1959, 2003.

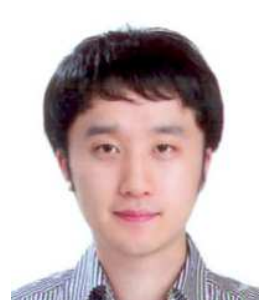

Hyung-Gu Park received his B.S. degree from the Department of Electronic Engineering at Konkuk University, Seoul, Korea, in 2010, where he is currently working toward the Ph.D. degree in College of Information and Communication Engineering, Sungkyunkwan University. His research interests include high-speed interface IC and CMOS RF transceiver.

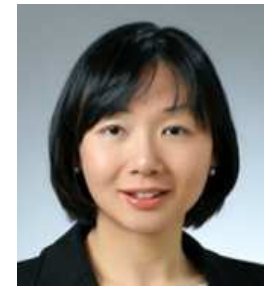

SoYoung Kim received a B.S. degree in Electrical Engineering from Seoul National University, Seoul, Korea in 1997 and M.S. and Ph.D. degrees in Electrical Engineering from Stanford University, Stanford, CA in 1999 and 2004, respectively. From 2004 to 2008, she was with Intel Corporation, Santa Clara, CA, where she worked on parasitic extraction and simulation of on-chip interconnects. From 2008 to 2009, she was with Cadence Design Systems, San Jose, CA, where she worked on developing IC power analysis tools. She is currently an Assistant Professor with the College of Information and Communication Engineering, Sungkyunkwan University, Suwon, Korea. Her research interests include device and interconnect modeling, signal integrity, power integrity and electromagnetic interference in electronic systems.

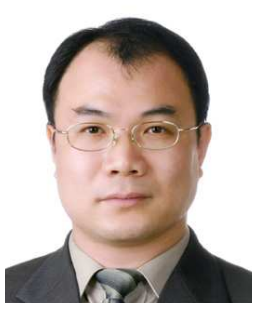

Kang-Yoon Lee received the B.S., M.S. and Ph.D. degrees in the School of Electrical Engineering from Seoul National University, Seoul, Korea, in 1996, 1998, and 2003, respectively. From 2003 to 2005, he was with GCT Semiconductor Inc., San Jose, CA, where he was a Manager of the Analog Division and worked on the design of CMOS frequency synthesizer for CDMA/PCS/PDC and single-chip CMOS RF chip sets for W-CDMA, WLAN, and PHS. From 2005 to 2011, he was with the Department of Electronics Engineering, Konkuk University as an Associate Professor. Since 2012, he has been with College of Information and Communication Engineering, Sungkyunkwan University, where he is currently an Associate Professor. His research interests include implementation of power integrated circuits, CMOS RF transceiver, analog integrated circuits, and analog/digital mixed-mode VLSI system design. 